\title{
Efficacy of Topical Heparin in Preventing the Incidence of Thrombophlebitis After Peripheral Intra Venous Cannulation.
}

\author{
Dr. Omais Ali Beigh ${ }^{1}$ dr. Habib Rahaman ${ }^{2}$ \\ ${ }^{1}$ Post Graduate Department Of Anaesthesiology, Yenepoya Medical College, Deralakatte, Mangalore \\ ${ }^{2}$ Assosiate Professor Department Of Anaesthesiology, Yenepoya Medical College, Deralakatte, Mangalore.
}

\begin{abstract}
A common complication associated with Intra Venous therapy is Phlebitis. Numerous literature articles have written that emphasize that phlebitis can occur in more then $50 \%$ of patients receiving IV therapy. It is essential to be able to identify who are at risk of developing thrombophlebitis. Heparin reduces the superficial thrombophlebitis. This study was conducted at yenepoya medical college hospital The patients were divided into two groups, using closed envelope method. Group $H$ included 70 patients who received topical heparin and Group C included 70 patients who did not receive topical heparin. The patients were studied for the results on the basis of Phlebitic scale at the intervals of 0 hours, 12 hours, 24 hours, 48 hours and 72 hours. the mean age was calculated as 33.94 There was significantly lesser score in group $H$ (receiving topical heparin) in all time periods with $p$ value of <0.001.and we concluded that prophylaxis with topical heparin is useful in managing a patient with intravenous cannula.
\end{abstract}

Keywords: Topical Heparin, Thrombophlebitis , Intra Venous Cannulation

\section{Introduction}

A common complication associated with Intra Venous ( IV) therapy is Phlebitis. Numerous literature articles have written that emphasize that phlebitis can occur in more then $50 \%$ of patients receiving IV therapy. Phlebitis is defined as the acute inflammation of the internal lining of the vein. Phlebitis is characterized by pain and tenderness along the course of the vein. Redness, swelling and warmth can be felt at the insertion site. Peripheral catheter related phlebitis, is caused by the inflammation of tunica intima of a superficial vein. The inflammation is due to irritation of the tunica intima by mechanical, chemical or bacterial sources. If felt untreated, it can lead to infection or thrombus formation. It is essential to be able to identify who are at risk of developing thrombophlebitis. In turn, early recognition will enable prompt intervention. Heparin which is a non uniform mixture of strait chain mucopolysaccharides reduces the superficial thrombophlebitis.

\section{Materials and methods}

This study was conducted by the Department of Anesthesiology at YENEPOYA MEDICAL College Hospital over a period of three months from December 2015 to February 2016 after getting ethical clearance from Yenepoya University Ethics Committee. A randomized observational study was conducted on 140 patients posted for surgery in which IV antibiotics and Fluids are expected to be administered for more than 72 hours as well as patients admitted in the ICU, belonging to age group of 14 to 55 years. The patients were divided into two groups, using closed envelope method. Group $\mathrm{H}$ included 70 patients who received topical heparin and Group C included 70 patients who did not receive topical heparin. Statistical Analysis was based on Independent ${ }^{\prime}$ ' test (or Mannwhitney non parametric test for two independent groups) with power 0.8 , effect size 0.5. Sample size was calculated using the statistical software $\mathrm{G}^{*}$ power 3.0.10.

Patients in the OT or ICU with a good peripheral vein on the forearm away from the joints were selected. Area was cleaned with surgical spirit and an 18 Gauge Cannula (B Braun Vasofix) was inserted using aseptic precaution. After insertion of the cannula and before securing the cannula topical heparin $1000 \mathrm{IU} / \mathrm{ml}$ (Phlebotroy QPS, Troika Pharmaceuticals) 10 drops were applied to the said site along the length of the cannula. Then the site was covered by adhesive tape (3M micro pore) and that time was marked as ' $\mathrm{O}$ ' hours. Every 12 hours the same site was examined using Phlebotic scale and again 10 drops of $1000 \mathrm{IU}$ of topical Heparin was applied and site was again covered by adhesive tape for 72 hours. Patients were advised to avoid hand washing. The data collection tool was designed and validated by the research group based on the theory and guidelines on phlebitis, after it had been tested in a clinical setting. Phlebitis scale proposed by the RCN 2010. Patients not giving consent, with known hypersensitivity, coagulation disorders or on anticoagulants were not included in the study. 


\section{Results and observations}

In this study a total of 140 patients undergoing intra venous cannulation were divided into two groups with 70 patients in each group. A total of 70 patients in $\mathrm{H}$ group (patients receiving topical heparin) and 70 in $\mathrm{C}$ group (patients not receiving topical heparin). The patients were studied for the results on the basis of Phlebitic scale at the intervals of 0 hours, 12 hours, 24 hours, 48 hours and 72 hours. The result was calculated by using Chi Square test at each time period as shown in tables 1, 2 and 3. It was observed that there is significantly lesser Phlebitis score in Group H (patients receiving topical heparin) as compared to Group C (patients not receiving heparin). Also we observed the frequency of incidence in different age groups as shown in tables 4,5 and 6. In our study the mean age was calculated as 33.94 and it was seen that the incidence is more in male patients.

\begin{tabular}{|l|l|l|l|l|l|l|l|}
\hline & GROUP & N & Mean & Std. Deviation & t & df & P VALUE \\
\hline \multirow{2}{*}{ AGE } & GROUP C & 70 & 35.69 & 11.348 & 2.028 & 130.75 & $\mathbf{0 . 0 4 5}$ \\
\cline { 2 - 6 } & GROUP H & 70 & 32.19 & 8.927 & & & \\
\hline
\end{tabular}

Table 1: Age distribution in the study

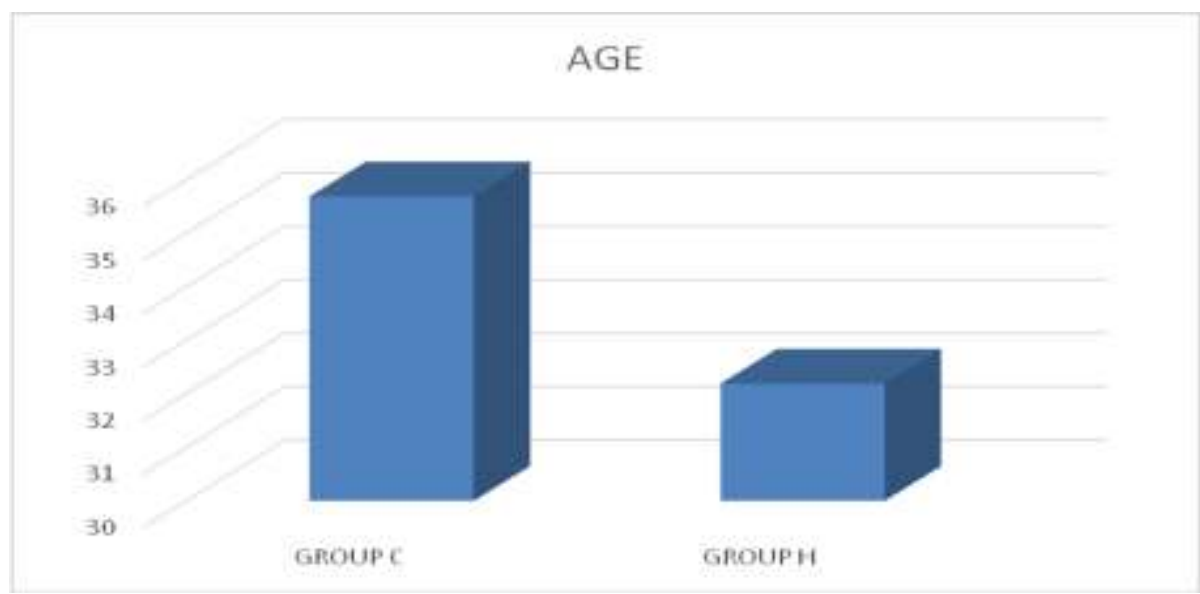

Graph 1: Age distribution in the study

\begin{tabular}{|c|c|c|c|}
\hline \multicolumn{4}{|c|}{ GENDER } \\
\hline & & Frequency & Percent \\
\hline \multirow[t]{3}{*}{ Valid } & $\mathrm{F}$ & 63 & 45.0 \\
\hline & M & 77 & 55.0 \\
\hline & Total & 140 & 100.0 \\
\hline
\end{tabular}

Table 2: Gender distribution in the study

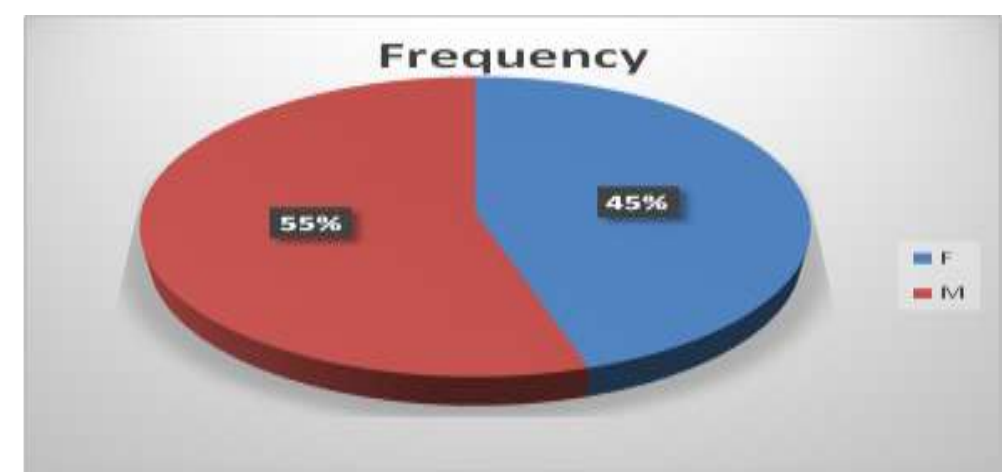

Graph2: Gender distribution in the study

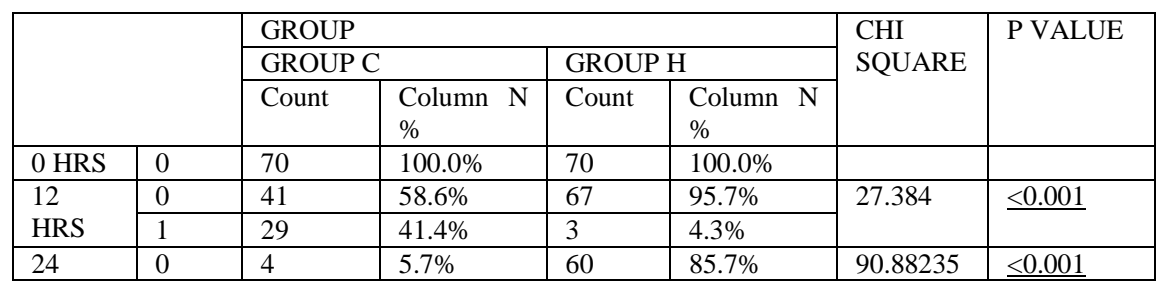


Efficacy Of Topical Heparin In Preventing The Incidence Of Thrombophlebitis After Peripheral...

\begin{tabular}{|c|c|c|c|c|c|c|c|}
\hline \multirow[t]{2}{*}{ HRS } & 1 & 58 & $82.9 \%$ & 10 & $14.3 \%$ & & \\
\hline & 2 & 8 & $11.4 \%$ & 0 & $0.0 \%$ & & \\
\hline \multirow{3}{*}{$\begin{array}{l}48 \\
\text { HRS }\end{array}$} & 0 & 0 & $0.0 \%$ & 34 & $48.6 \%$ & \multirow{3}{*}{63.16571} & \multirow[t]{3}{*}{$<0.001$} \\
\hline & 1 & 26 & $37.1 \%$ & 30 & $42.9 \%$ & & \\
\hline & 2 & 44 & $62.9 \%$ & 6 & $8.6 \%$ & & \\
\hline \multirow{3}{*}{$\begin{array}{l}72 \\
\text { HRS }\end{array}$} & 1 & 3 & $4.3 \%$ & 44 & $62.9 \%$ & \multirow[t]{3}{*}{61.80705} & \multirow[t]{3}{*}{$<0.001$} \\
\hline & 2 & 47 & $67.1 \%$ & 26 & $37.1 \%$ & & \\
\hline & 3 & 20 & $28.6 \%$ & 0 & $0.0 \%$ & & \\
\hline
\end{tabular}

Table 3: Phlebitic scale at the intervals of 0 hours, 12 hours, 24 hours, 48 hours and 72 hours

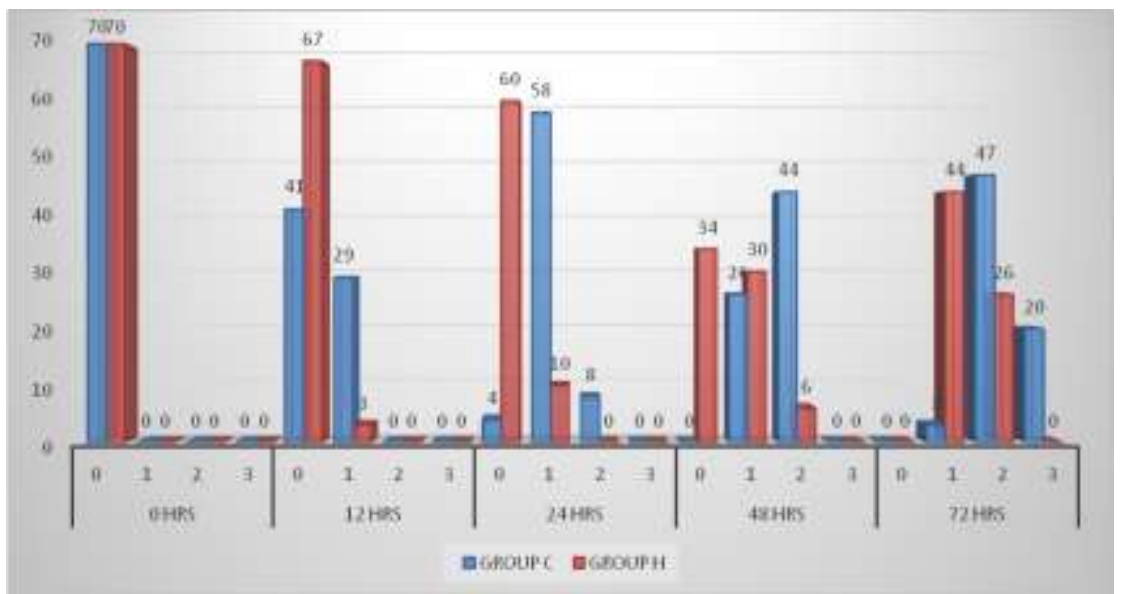

Graph 3: Phlebitic scale at the intervals of 0 hours, 12 hours, 24 hours, 48 hours and 72 hours

\section{Discussion}

Phlebitis is the most common complication of IV therapy and several factors has been implicated in its pathogenesis. Chemical factors such as irritant drugs and physical factors such as duration of cannulation are just few of the identified risk factors for the development of phlebitis.

We graded phlebitis observed using a scale adapted from the clinical grading of phlebitis by infusion nursing society (2006). Mild grades of phlebitis was the commonest, comprising approximately two thirds of the observed incidence. Moderate and severe phlebitis were uncommon. It is important to note that most cases of phlebitis were detected and then preventive measures taken before moderate to severe thrombophlebitis developed but in our study we use topical heparin prophylactically to prevent or reduce the incidence.

The peripheral vein is traumatized during insertion of peripheral venous catheter. The catheter is also foreign to the human body. This initial trauma and presence of foreign body in the vein stimulates an inflammatory response which predisposes the development of thrombus and subsequent phlebitis. Catheters that are left longer in situ also have increased exposure to handling and drugs infusion which may explain the higher rate of thrombophlebitis for longer duration of its insertion.

Both genders are identified as a significant risk factor to the development of thrombophlebitis secondary to peripheral venous catheterization. A catheter that is used for infusion has double the risk of developing thrombophlebitis compared to a catheter that has not been used. This may be due to the type of solution infused through the catheter. Certain infusates such as antibiotics, chemotherapeutic drugs, solutions of low $\mathrm{pH}$ and high osmolarity are associated with increased risk of thrombophlebitis. We attempted to observe for an increased incidence of thrombophlebitis when infusate are used in patients. However, there was no significant difference between type of infusate and the development of thrombophlebitis among the sample population in this study. This may be due to the inadequate size of the study sample and the fact that most of the patients received different infusate through the same peripheral catheter. A further study using only one infusate per catheter may be helpful in confirming the risk of different infusates on development of thrombophlebitis.

For difference in risk and incidence of thrombophlebitis in different age groups, it was found that the incidence of thrombophlebitis was similar throughout all age groups of patients. The age of a patient did not influence the development of thrombophlebitis among our patients. This observation has also been reported in other studies.

The duration a catheter is left in the vein was found to significantly influence the incidence of thrombophlebitis. Patients who have a catheter for more than 3 days are more likely to have an increased risk of developing thrombophlebitis. Similar findings have previously been reported by Uslusoy and Barker. The duration of catheterization is the only modifiable risk factor identified. The results of many studies have shown that the risk of thrombophlebitis increases with increased duration of catheterization. It is recommended that prophylaxis resitting of catheter should be practised in all patients. The catheter should be removed or replaced in a different 
site after 72 hours of insertion, even when there is no sign of thrombophlebitis. The practice of electively replacing venous catheter after 72 hours should therefore be practised for all patients. Catheters which are no longer required should also be removed .

The choice of catheter size inserted is influenced by the rate of infusion required in the management of patient. Patients anticipated requiring rapid infusion of fluids or blood products will have larger catheters inserted. The choice of catheter size should therefore be made based on the rates of infusion needed for the patient.

The location of catheter placement has been found by previous studies to influence the incidence of peripheral venous catheter thrombophlebitis. Catheters placed on the lower limbs have been found to have higher incidence than catheters in the veins of the upper limb. On the upper limb, the brachium is preferred to veins on the dorsum of the hand due to a lower risk of developing thrombophlebitis. We were not able to study the difference in incidence rate between catheters placed in veins of upper and lower limbs as only one patient had a catheter in the lower limb. It is a standard practice in our hospital to avoid placing catheters in the lower limb unless unavoidable.

In this study, the incidence of thrombophlebitis between placement of catheter in veins on the dorsum and brachium of the upper limb was similar among patients for both sites. However, it should be noted that only a small number of the patients had catheters placed in the brachial veins and this may have influenced observed findings in this study. Placement of catheter on brachium veins is superior to dorsal veins and upper limb is preferable to veins of the lower limbs.

We would like to recommend that all patients with peripheral vein catheter in situ be screened for complications of peripheral venous catheter at least once daily. This should be performed by visual examination and palpation of the vein for warmth, tenderness, erythema and a palpable cord. Patients with these symptoms should have their catheters replaced at a different site. Malfunctioning catheters should also be replaced. We propose that all units should have an observation chart to document development of signs of thrombophlebitis. The chart should include the signs mentioned as well proper documentation of the date of catheterisation. This would help detect thrombophlebitis much earlier and decrease patients' discomfort and pain. Catheters that are not used should be removed within 72 hours of placement or when signs of developing thrombophlebitis have been detected.

Among the 140 subjects both in the control and heparin group evaluated in this study, significantly more patients in the control group developed phlebitis thereby giving an high incidence rate. The results therefore suggests that applying topical heparin in the IV insertion site may the decrease the risk of phlebitis. The length of time that the IV cannula is in place is traditionally believed to be directly correlated with the incidence of phlebitis.

\section{Conclusion}

In our study we concluded that if prophylaxis with topical heparin is useful managing a patient with intravenous cannula in order to reduce the high incidence of thrombophlebitis associated with intravenous cannula .

\section{Acknowledgements}

An acknowledgement section may be presented after the conclusion, if desired.( 8)

\section{References}

[1]. Endacott R et al (2009) Clinical Nursing Skills, Core and Advanced. Oxford: Oxford University Press

[2]. Uslusoy E,Mete S, predisposing factors for the development of thrombophlebitis with peripheral IV cannulas a descriptive study ,2008Apr;20(4):172-180.

[3]. Macklin D (2003) Phlebitis, a painful complication of peripheral IV catheterization that may be prevented. American Journal of Nursing; 103: 2,55-60.

[4]. Kohno E et al (2009) Effects of corticosteroids on phlebitis induced by intravenous infusion of antineoplastic agents in rabbits. International Journal of Medical Sciences; 6: 4, 218-223.

[5]. Catton JA et al (2006) The effect of heparin in peripheral intravenous nutrition via a fine-bore

[6]. midline: a randomised double-blind controlled trial.Clinical Nutrition; 25: 394-399.

[7]. Malach T et al (2006) Prospective surveillance of phlebitis associated with peripheral intravenous catheters. American Journal of Infection Control;34: 5, 308-312.

[8]. LaRue G, Peterson M (2011) The impact of dilution on intravenous therapy. Journal of Infusion Nursing: 34, 2, 117-123.

[9]. Kuwahara T et al (1999) Experimental infusion phlebitis: tolerance pH of peripheral vein. Journal of Toxicological Sciences; 24: 2, 113-121.

[10]. Hankins J, Waldman Lonsway RA, Hedrick C, Perdue MB. The Infusion Nurses Society. Infusion Therapy in Clinical Practice. 2nd Edition, 2001. Ch. 8 Infection Control, 131-132.

[11]. Karadag A, Gorgulu S. Journal of IV Nursing. Effect of two short peripheral catheter materials on phlebitis development. 200, May/June, 23:3, 158-166.

[12]. Maki D., Ringer, M. Annals of Internal Medicine. Risk factors for infusion related phlebitis with small peripheral venous catheters: a randomised trial. 1991, 114:10, 845-54.

[13]. Mazzola, JR, Schott- Baer, D, Addy L. Journal of IV Nursing. Clinical Factors Associated with the development of phlebitis after insertion of a peripherally inserted central catheter. 1999, January/February, 22:1, 36-42. 
[14]. Curran, E., Coia, J., Gilmour, H., McNamee, S., and Hood, J. 2000. Multi-centre research surveillance project to reduce infections/phlebitis associated with peripheral vascular catheters. Journal of Hospital Infection, 46(3):194-202.

[15]. Homer LD, Holmes KR, Risk associated with $72 \mathrm{hrs}$ and 96 hrs IV catheter dwell times, Journal of IV nursing,1998 October; 21(5): 301-305.

[16]. Doris A Millam, Susan Masoorly, Avoiding the pit falls of IV therapy, The Nurse Friendly 2004; $1(15) ; 9$.

[17]. Kerrison T and Woodhull J (1994), Reducing the risk of thrombophlebitis, Professional Nurse, 9(10), 662-666.

[18]. Singh R, Bhandary S , peripheral IV catheters related thrombophlebitis and its contributing factors among adult population in a teaching hospital, 2008 Oct -Dec;6(24):443-447

[19]. Tagalakis V ,Kahn SR,Libman M ,The epidemiology of peripheral vein infusion thrombophlebitis, a critical review ,2002Aug1;113(2):146-151

[20]. Cornely OA,Bethe U ,Pauls R ,peripheral Teflon catheters :factors determining incidence of phlebitis and duration of cannulation,2002May;23(5):249-253.

[21]. Monreal M,Oller B,Rodriguez N et al, infusion phlebitis in postoperative patient: when and why, 1999;29(5) 247-254

[22]. Subramanyam M (1989), IV placement devices and infusion thrombophlebitis, Indian Journal of medical sciences, 43(2), $27-31$.

[23]. Horwood K, R Abraham, Complications and risk factors in IV therapy, Journal of IV nursing, 1999, 5(2):2. 